\title{
MINIMUM ENTROPY PRODUCTION PRINCIPLE AND OSTWALD RIPENING ह2.
}

\section{Scientific Report}

\section{G. C. Kuczynski}

University of Notre Dame Notre Dame, IN 46556

February, 1978

Prepared for

THE U. S. DEPARTMENT OF ENERGY

UNDER CONTRACT NO. EG-77-S-02-4385. 


\section{DISCLAIMER}

This report was prepared as an account of work sponsored by an agency of the United States Government. Neither the United States Government nor any agency Thereof, nor any of their employees, makes any warranty, express or implied, or assumes any legal liability or responsibility for the accuracy, completeness, or usefulness of any information, apparatus, product, or process disclosed, or represents that its use would not infringe privately owned rights. Reference herein to any specific commercial product, process, or service by trade name, trademark, manufacturer, or otherwise does not necessarily constitute or imply its endorsement, recommendation, or favoring by the United States Government or any agency thereof. The views and opinions of authors expressed herein do not necessarily state or reflect those of the United States Government or any agency thereof. 


\section{DISCLAIMER}

Portions of this document may be illegible in electronic image products. Images are produced from the best available original document. 


\title{
MINIMUM ENTROPY PRODUCTION PRINCIPLE AND OSTWALD RIPENING
}

\author{
G. C. Kuczynski \\ Department of Metallurgical Engineering \\ and Materials Science \\ University of Notre Dame. \\ Notre Dame, Indiana
}

\section{INTRODUCTION}

The problem of growth of particles in a medium capable of transmitting matter has been a subject of several theoretical treatments. $1,2,3$ All of them have tried to find a steady state solution to a continuity equation containing a distribution function for particle size, coupled with an expression for rate of change of particle size, usually obtained from a mean field approximation. No attempt has been made to derive the distribution function from a more general principle.

The objective of this note is to demonstrate that a distribution function can be obtained from a general principle such as the principle of minimum rate . of entropy production. 4 


\section{GENERAL RELATIONS}

Let us consider $\mathrm{N}_{\mathrm{v}}$ spherical particles of phase $\beta$ dispersed in a phase $\alpha$. $\mathrm{N}_{\mathrm{v}}$ is a function of time only. The distribution function of radii of the particles $f(r, t)$ is defined as $f(r, t) d r$ being the number of particles having radius between $r$ and $r+d r$, at a time $t$, per unit volume. The interaction among the particles is expressed by a known rate of the change of the particle radius with time, $\frac{d r}{d t}=v(r, t)$. The continuity equation can be written in the form

$$
\frac{\partial f}{\partial r}+\frac{\partial(f v)}{\partial r}=0
$$

We can normalize function $f(r, t)$ by introducing function $p(r, t)=f(r, t) / N_{v}$. Obviously

$$
\int_{r_{c}}^{\infty} f(r t) d r=N_{v} \text { and } \int_{r_{c}}^{\infty} p(r, t) d r=1
$$

where $r_{c}$ is the radius of the smallest aggregate of atoms which can be still considered as a particle of the phase $\beta$ and not a fluctuation in the matrix $\alpha$. This problem will be discussed later in more detail. Equation (1) can be rewritten in the form:

$$
\frac{\partial p}{\partial t}=-\frac{d{ }^{n N}}{d t} p-\frac{\partial(v p)}{\partial r}
$$

From (2) it is obvious that

$$
\int_{r_{c}}^{\infty} \frac{\partial f(r t)}{\partial t} d r=\dot{N}_{v}=\frac{d N}{d t} \text { and } \int_{r_{c}}^{\infty} \frac{\partial p(r t)}{\partial t} d r=0
$$

Integrating both sides of (3) we obtain:

$$
\frac{\mathrm{d} \operatorname{lnN} v}{\mathrm{dt}}=-\int_{\mathrm{r}_{\mathrm{c}}}^{\infty} \frac{\partial(\mathrm{vp})}{\partial \mathrm{r}} \mathrm{dr}=\mathrm{v}\left(\mathrm{r}_{\mathrm{c}}\right) \mathrm{p}\left(\mathrm{r}_{\mathrm{c}}\right)
$$




\section{RATE OF ENTROPY PRODUCTION}

Turning now to the rate of entropy production, we note that there are three sources of entropy: the first, the configurational entropy due to the change of distribution of particle size of the system, the second is due to the concentration gradient caused by capillary forces acting near the particle interfaces, and the third, to the reduction of total surface energy of the s.ystem.

The entropy $s_{1}$ per unit volume due to particle distribution given by function $\mathrm{p}$ is:

$$
\mathrm{S}_{1}=-\mathrm{N}_{\mathrm{v}} \mathrm{K} \int_{\mathrm{r}_{\mathrm{c}}}^{\infty} \mathrm{p} \ln \mathrm{p} \mathrm{dr}
$$

and the rate of this entropy increases

$$
\frac{d S_{1}}{d t}=-N_{v} k \int_{r_{c}}^{\infty} \frac{\partial p}{\partial t}(1+\ln p) d r
$$

which in view of the second equation (4) is

$$
\frac{d S_{1}}{d t}=-N_{v} k \int_{r_{c}}^{\infty} \frac{\partial p}{\partial t} \ln p d r
$$

The rate of entropy production due to the chemical potential $\mu$ :gradient around one particle of radius $r$ per unit volume ${ }^{4}$ is:

$$
\frac{d \cdot S_{r}}{d t}=-\frac{1}{T} \int_{r}^{\infty} J \frac{\partial \mu}{\partial x} 4 \pi x^{2} \cdot d x
$$

where $J$ the diffusion $f$ lux is given by the familiar relation

$$
J=-B \frac{\partial \mu}{\partial x}
$$

and mobility $B=\frac{D C}{K T}$, where $D$ is diffusion coefficient and $C_{0}$ the concentration expressed as the number of atoms or vacancies per unit volume, away from the 
particle interface. Inserting (9) into (8) we obtain

$$
\frac{d S_{r}}{d t}=4 \pi \frac{D C}{k T^{2}} \cdot \int_{r}^{\infty}\left(\frac{\partial \mu}{\partial x}\right)^{2} x^{2} d x
$$

Chemical potential of a solution assumed to be ideal is:

$$
\mu=\mu_{0}+k T \ln C
$$

and

$$
\frac{\partial \mu}{\partial x}=\frac{k T}{C} \frac{\partial C}{\partial x}
$$

If the particle is a sphere then the steady state solution of the diffusion equation yields:

$$
c=\frac{a}{x}+b
$$

where constants $a$ and $b$ are determined from the boundary conditions

$$
\begin{aligned}
& x=r, C=C_{0}+\Delta C \\
& x \rightarrow \infty, C \rightarrow C_{0}
\end{aligned}
$$

Thus

$$
c=c_{0}+\frac{r \Delta C}{x}
$$

and

$$
\frac{\partial C}{\partial x}=-\frac{a}{x^{2}}=-\frac{r \Delta C}{x^{2}}
$$

where $\quad \Delta c=\frac{2 C_{0} \gamma \Omega}{k T r}$

$\gamma$ being the interface tension and $\Omega$ the atomic volume.

This relation is true when the radius of a particle is not too small. For $\frac{2 \gamma \Omega}{\mathrm{kTr}} \gg 1$

$$
\Delta \mathrm{C}=\mathrm{C}_{0} \cdot\left[\exp \left(\frac{2 \gamma r}{\mathrm{kTr}}\right)-1\right]
$$

For simplicity we shall use relation (15) throughout. Introducing (14) first to (11') and then (11') into (10) and integrating, 
we obtain

$$
\frac{\mathrm{d}^{\mathrm{S}} \mathrm{r}}{\mathrm{dt}}=-\frac{4 \pi \mathrm{DC} \mathrm{o}^{2}}{\mathrm{~T}}\left(\frac{\Delta \mathrm{CkT}}{\mathrm{C}_{0}}\right)^{2}\left[\frac{1}{\mathrm{x}}\right]_{\mathrm{r}}^{\infty}=\frac{4 \pi \mathrm{Dr}}{\mathrm{C}_{\mathrm{o}}} \frac{(\Delta \mathrm{CkT})^{2}}{\mathrm{~T}}
$$

Replacing $\Delta C$ by expression (15) and rearranging

$$
\frac{d_{1}{ }^{S}}{d t}=\frac{16 \pi D C_{0}(\gamma \Omega)^{2}}{r T}
$$

Therefore the rate of entropy production per unit volume due to the gradients near $\mathrm{N}_{\mathrm{v}}$ particles is

$$
\frac{d S_{2}}{d t}=N_{v} \int_{r_{c}}^{\infty} \frac{d^{S} r_{p}}{d t} \mathrm{dr}=16 \pi N_{v} k_{0}\left(\frac{\gamma \Omega}{k T}\right)^{2}\left(\frac{\overline{1}}{r}\right) .
$$

where a bar over a function signifies its mean value.

This equation is approximately true provided that $\mathrm{N}_{\mathrm{v}}$ is not too large and the overlaps of concentration can be neglected.

Finally, the third source of entropy is the reduction of the total particle area. The rate of entropy production in this case can be calculated from the deDonder theorem, 4

$$
\frac{d S_{3}}{d t}=-\frac{1}{T} \frac{d \Delta G}{d t}
$$

where $\Delta G$ is the Gibbs free energy of the system per unit volume. In our case $\Delta G=\gamma A$, where $A$ is the total particle area per unit volume. Equation (19) can be rewritten in the form

$$
\frac{\mathrm{dS}_{3}}{\mathrm{dt}}=-\frac{\gamma}{\mathrm{T}} \frac{\mathrm{dA}}{\mathrm{dt}}
$$

which with the help of A6 becomes

$$
\frac{\mathrm{dS}_{3}}{\mathrm{dt}}=-\frac{2 \mathrm{~N}_{\mathrm{V}} \mathrm{T}}{\mathrm{T}} \overline{\mathrm{rv}}
$$

The total rate of entropy production is obtained by adding ( $\left.7^{\prime}\right),(18)$ and (21) 


$$
\frac{d S}{d t}=\frac{d S_{1}}{d t_{2}}+\frac{d S_{2}}{d t}+\frac{d S_{3}}{d t}=-N_{v} k\left\{\int_{r_{c}}^{\infty}\left(\frac{\partial p}{\partial t} \ln p-\frac{M}{r}+\frac{2 \gamma}{k T} r v\right) p d r\right\}
$$

where $M=16 \pi \mathrm{DC}{ }_{0}\left(\frac{\gamma \Omega}{\mathrm{kT}}\right)^{2}$.

\section{DERIVATION OF DISTRIBUTION FUNCTION}

Now the function in equation (19) must be minimized with respect to all possible distribution functions, $p(r, t)$, subject however to certain conservation conditions, the first of which is that

$$
\int_{r_{c}}^{\infty} \operatorname{pdr}=1 \text {. }
$$

One would be tempted to. assume $\overline{\mathbf{r}^{2} v}=0$ which means that the total volume of precipitates is conserved. However, as particles grow, the excess concentration around them decreases and in fact $\overline{r^{2} v}>0$. This problem is discussed in detail in Appendix $B$, where also it is shown that

$$
\overline{\mathrm{r}^{2} \mathrm{v}}=K_{1}\left(\frac{\overline{\partial \mathrm{v}}}{\partial \mathrm{r}}\right)
$$

where. $\quad \mathrm{K}_{1}=\frac{4 \pi \mathrm{C}_{0} \gamma \Omega \mathrm{L}^{2}}{\left(\mathrm{C}_{1}-\mathrm{C}_{0}\right) \mathrm{kT}}$.

Therefore as the second conservation condition we have

$$
\int_{c}^{\infty}\left(r^{2} v-K_{1} \frac{\partial v}{\partial r}\right) p d r=0
$$

Finally we have to express the obvious fact that

$$
\frac{d \ln N_{v}}{d t}<0
$$

Let us take equation (3) and rewrite it in the form

$$
\frac{\partial p}{\partial t}=-\frac{d \operatorname{lnN} v}{d t} p-\frac{\partial v}{\partial r} p-v \frac{\partial p}{\partial r}
$$


But the last term on the right side is simply $\frac{\partial p}{\partial t}$ because

$$
v \frac{\partial p}{\partial r}=\frac{v}{\left(\frac{d r}{d t}\right)} \frac{\partial p}{\partial t}=\frac{\partial p}{\partial t}
$$

Therefore

$$
2 \frac{\partial p}{\partial t}=-\frac{d l n N}{d t} p-\frac{\partial v}{\partial r} p
$$

Integrating both sides and taking into consideration (4) we obtain

$$
\frac{\overline{\partial v}}{\partial r}=-\frac{d \ln N}{d t}
$$

Hence $\left(\frac{\partial v}{\partial r}\right)$ is always positive.

Therefore another conservative condition is

$$
\left.\int_{r}^{\infty}\left(\frac{\partial v}{\partial r}\right)-\frac{\overline{\partial v}}{\partial r}\right) p d r=0
$$

To minimize function (22) variational calculus is used. First let us find a function I independent of conditions (2), (23) and (28). Using the method of Lagrange multipliers we multiply equation (2) by $\left(-\mathrm{N}_{\mathrm{v}} \mathrm{kn}\right),(23)$ by $\left(-\mathrm{N}_{\mathrm{v}} \mathrm{k} \beta\right)$ and (28) by $\left(-\mathrm{N}_{\mathrm{v}} \mathrm{k} \lambda\right)$ and add the products to the function under the integral in equation (19). The desired function $I$ has the form

$$
\begin{aligned}
& I=\frac{\partial p}{\partial t} \operatorname{lnp} \cdot p+n p+\frac{2 \gamma}{k T} r v-\beta K_{1} \frac{\partial v}{\partial r} p-\lambda \frac{\partial v}{\partial r} p+\beta^{2} r^{2} v p-\frac{M}{r} p+\lambda \frac{\partial v}{\partial r} p \\
& \text { or, using (3) and (27), } \\
& I=\left(\frac{\partial v}{\partial r}\right) \operatorname{lnp} \cdot p-\frac{\partial v}{\partial r} \operatorname{lnp} \cdot p-v \operatorname{lnp} \frac{\partial p}{\partial r}+\eta p+\frac{2 k}{k T} r v-\beta K_{1} \frac{\partial v}{\partial r} p \\
& \quad-\lambda \frac{\partial v}{\partial r} p+\beta^{2} r^{2} v p-\frac{\mu}{r} p+\lambda \frac{\partial v}{\partial r} p .
\end{aligned}
$$

The solution of the variational equation

$$
\delta \int_{r_{c}}^{\infty} \mathrm{I} \mathrm{dr}=\dot{0}
$$


or, equivalently,Euler's equation

$$
\frac{\partial I}{\partial p}-\frac{d}{d r}\left(\frac{\partial I}{\partial p_{r}}\right)=0
$$

with $p_{r}=\frac{\partial p}{\partial r}$, should yield a distribution function $p(r, t)$ which minimizes the rate of entropy production given by equation (22);

$$
\begin{gathered}
\frac{\partial I}{\partial p}=\left(\frac{\partial v}{\partial r}\right)+\left(\frac{\partial v}{\partial r}\right) \operatorname{lnp}-\frac{\partial v}{\partial r}-\frac{\partial v}{\partial r} \operatorname{lnp}-v \frac{\partial \operatorname{lnp}}{\partial r}+\frac{2 \gamma}{k T} r v+ \\
n-\beta K_{1} \frac{\partial v}{\partial r}-\lambda\left(\frac{\partial \bar{v}}{\partial r}\right)+\beta r^{2} v-\frac{M}{r}+\lambda \frac{\partial v}{\partial r} \\
\frac{\partial I}{\partial p_{r}}=-v \operatorname{lnp} \text { and }-\frac{d}{d r}\left(\frac{\partial I}{\partial p_{r}}\right)=\frac{\partial v}{\partial r} \operatorname{lnp}+v \frac{\partial \ln p}{\partial r}
\end{gathered}
$$

Euler's equation (32) becomes

$$
\left(\frac{\overline{\partial v}}{\partial r}\right) \operatorname{lnp}-(\lambda-1)\left(\frac{\partial v}{\partial r}\right)+n+\beta r^{2} v+\frac{2 \gamma}{k T} r v-\frac{M}{r}+\left(\lambda-\beta K_{1}-1\right) \frac{\partial v}{\partial r}=0
$$

Yielding expression for $p(r t)$ in the form

$$
\left(\frac{\partial v}{\partial r}\right) \ln p=\eta+(\lambda-1)\left(\frac{\partial v}{\partial r}\right)-\beta r^{2} v-\frac{2 \gamma}{k T} r v+\frac{M}{r}-\left(\lambda-\beta K_{1}-1\right) \cdot \frac{\partial v}{\partial r}
$$

$\left(\frac{\partial v}{\partial r}\right)$ Inp is always negative because $p(r, t)<1$. For very large particles $p(r t)$ should approach zero hence $\operatorname{lnp} \rightarrow-\infty$. Also for large particles $\frac{M}{r}$ and $\frac{\partial v}{\partial r}$ approach zero, hence $\beta r^{2} v \rightarrow \infty$ and $\beta$ must be positive.

'For small particles as $r \rightarrow r_{c}$ only the last two terms on the right side of equation (30) count. The condition that the right side is negative is

$$
-\frac{2 \gamma}{k T} r_{c} v\left(r_{c}\right)+\frac{M}{r_{c}}-\left(\lambda-\beta K_{1}-1\right)\left(\frac{\partial v}{\partial r}\right)_{r_{c}}<0
$$

and as $\left(\frac{\partial v}{\partial r}\right)_{r_{c}}>0$

$$
\lambda>1+\beta K_{1}+\frac{M-\frac{2 \gamma}{k T} r_{c} v\left(r_{c}\right)}{r_{c}\left(\frac{\partial v}{\partial r}\right)_{r_{c}}}
$$


Summing up results obtained in this section one may say that the distribution function $p(r t)$ for small $r$ decreases to zero as an exponential function which can be approximated by an expression

$$
p(r t) \rightarrow \exp \left(-H(t) / r^{n}\right)
$$

where $H(t)$ is a function of time only and $n$ is a constant between 2 and 4 . At. large $r, p(r t)$ has an exponential tail. In between there must be some maximum which, in principle, could be determined from (33), setting $\frac{d \ln p}{d r}=0$. These properties of our distribution function are radically different from those obtained by Lifshitz and Slyozov ${ }^{2}$ and Wagner ${ }^{3}$ whose functions decrease to zero as $\dot{r}^{n}$ and have rather sharp cutoff at large particle radii. 
Appendix A

Mean Values

Evaluation of mean values of various functions introduced in the main text of this report is based on some simple theorems. which now will be derived.

$$
\overline{\frac{d r}{d t}}=\int_{r_{c}}^{\infty} r \frac{\partial p}{\partial t} d r
$$

which by equation (3) becomes

$$
\begin{aligned}
& \overline{\frac{d r^{n}}{d t}}=-\frac{d \operatorname{lnN} v}{d t} \overline{r^{n}}-\int_{r_{c}}^{\infty} r^{n} \frac{\partial\left(v_{p}\right)}{\partial r} d r \\
& =-\frac{d l n N}{d t} \overline{r^{n}}+r_{c}^{n} v\left(r_{c}\right) p\left(r_{c}\right)+n r^{\overline{n-1}}
\end{aligned}
$$

Dividing this equation by $\overline{r^{n}}$ and using equations (5) we obtain

$$
\frac{d \ln \overline{r^{n}}}{d t}=-\frac{d \operatorname{lnN} v}{d t}\left(1-\frac{r_{c}^{n}}{r^{n}}\right)+n \frac{\overline{r^{n-1} v}}{\overline{r^{n}}}
$$

or as $r_{c}{ }^{n} / \vec{r}$ is very small A3 can be written to good approximation

$$
\frac{d \ln \overline{r^{n}}}{d t}=\frac{d \ln N}{d t}+n \frac{\overline{r^{n-1} v}}{\overline{r^{n}}}
$$

This equation can be applied to obtain an expression for change of total area of particles per unit volume $A$ with time. By definition

$$
A=N_{v} \overrightarrow{r^{2}}
$$

Note that shape factor has been omitted. Converting A4 into logarithmic form and differentiating, we obtain 


$$
\frac{d \ln A}{d t}=\frac{d \operatorname{lnN} v}{d t} \cdot+\frac{d \ln \overline{r^{2}}}{d t}
$$

Combining A5 with A3', equation A5 takes the form

$$
\frac{d \ln A}{d t}=\frac{2 \overline{r v}}{\overline{r^{2}}}
$$

Next let us apply equation A3 to the evolution of total volume of precipitates or pores per untt volume in time. It is defined as

$$
\mathrm{p}=\mathrm{N}_{\mathrm{v}} \overline{\mathrm{r}^{3}}
$$

Again the shape factor is omitted. By the same mathematical manipulations as in the case of A given above we obtain

$$
\frac{d \ln P}{d t}=\frac{\overline{3 r^{2} v}}{\overline{r^{3}}}
$$

In case of precipitation of a second phase when total volume of molecules of that phase is conserved $\frac{d \ln P}{d t}=0$ and we have

$$
\overline{r^{2} v}=0
$$

This condition is only an approximation because some of the molecules are in supersaturated solution due to the capillary forces at the interfaces of small particles and are released slightly increasing $p$, as the particles grow.

Finally using equation $\mathrm{A}^{\prime}$ or $\mathrm{A}^{3}$ it is easy to demonstrate that

$$
\frac{d \bar{r}}{d t}-\bar{v}=-\frac{d \ln N_{v} \bar{r}}{d t}
$$

As $\frac{d \ln N}{d t}<0$ we conclude that

$$
\frac{d \bar{r}}{d t}>\bar{v}
$$


the difference $\frac{d \bar{v}}{d t}-\frac{\partial v}{\partial t}$ can be calculated as follows:

$$
\frac{\overline{\partial v}}{\partial t}=\int_{r_{c}}^{\infty} \frac{\partial v}{\partial t} p d r=\int_{r_{c}}^{\infty} \frac{\partial v}{\partial r} \frac{d r}{d t} p d r=\int_{r_{c}}^{\infty} v \frac{\partial v}{\partial r} p d r=\bar{v} \frac{\partial v}{\partial r}
$$

and

$$
\begin{aligned}
\frac{d \bar{v}}{d t}=\int_{r_{c}}^{\infty} \mathbf{v} \frac{\partial p}{\partial t} d r & =-\frac{d l n N}{d t} \bar{v}-\int_{r_{c}}^{\infty} v \frac{\partial(v p)}{\partial r} d r \\
& =\frac{d l n N}{d t}\left(v\left(r_{c}\right)-\bar{v}\right)+\bar{v} \frac{\partial v}{\partial r}
\end{aligned}
$$

Hence from $\mathrm{A} 12$ and $\mathrm{All}$

$$
\frac{d \bar{v}}{d t}-\frac{\bar{\partial}}{\partial t}=\frac{d \ln N}{d t}\left(v\left(r_{c}\right)-\bar{v}\right)
$$

As $\left|v\left(r_{c}\right)\right|>|\bar{v}|$, we have $\frac{d \bar{v}}{d t}<\frac{\overline{\partial v}}{\partial t}$. 


\section{APPENDIX B}

\section{Average Particle Volume Change}

It has been mentioned in the main text of this note that the relation $\overline{r^{2} v}$ should not be equal to zero. Indeed, it means that the particle volume is not conserved. In reality the total number of atoms is conserved. If before separation into two phases, the number of $B$ atoms per unit volume in homogeneous phase. is $C_{0}^{\prime}$ and after separation, $C_{0}$ in phase $\alpha$ and $C_{1}$ in $\beta$, then:

$$
C_{0}^{\prime}=\left(1-N_{v} \overline{2^{3}}\right) C_{0}+C_{1} N_{v} \cdot \overline{r^{3}}+\Delta N
$$

where $\Delta \mathrm{N}$ is the excess number of atoms $\mathrm{B}$ per unit volume maintained in $\alpha$ (matrix). near the curved interfaces of the dispersed particles. Let us first estimate a number $\Delta \mathrm{n}$ of $\mathrm{B}$ atoms near one particle of radius $r$

$$
\Delta n=4 \pi \int_{r}^{L}\left(C-C_{0}\right) x^{2} d x
$$

where $L$ is dimension of the specimen, $L \simeq 1$. But $C-C_{0}$ by equation (14). is $\mathrm{r} \Delta \mathrm{C} / \mathrm{x}$ where $\Delta \mathrm{C}=\frac{2 \mathrm{C}_{\mathrm{o}} \gamma \Omega}{\mathrm{kT}} \cdot$ Therefore

$$
\Delta \mathrm{n}=\frac{4 \pi \mathrm{C}_{\mathrm{o}} \gamma \Omega\left(\mathrm{L}^{2}-\mathrm{r}^{2}\right)}{\mathrm{kT}}
$$

and

$$
\Delta \mathrm{N}=\mathrm{N}_{\mathrm{v}} \int_{\mathrm{r}_{\mathrm{c}}}^{\infty} \Delta \mathrm{npdr}
$$

As $\mathrm{L} \gg \mathrm{r}$

$$
\Delta \mathrm{N}=\frac{4 \pi \mathrm{C}_{\mathrm{o}} \gamma \Omega \mathrm{N}_{\mathrm{v}} \mathrm{L}^{2}}{\mathrm{kT}}
$$

Inserting this into $\mathrm{B} 1$, differentiating both sides of the equation with respect to $t$, and rearranging, we obtain 


$$
0=\left(\frac{d \operatorname{lnN}{ }_{v}}{d t}+\frac{d \ln \overrightarrow{r^{3}}}{d t}\right) N_{v} \overrightarrow{r^{3}}\left(c_{1}-c_{0}\right)+\frac{4 \pi C_{o} \gamma \Omega L^{2} \dot{N}_{v}}{k T} \frac{d \operatorname{lnN} v}{d t}
$$

because obviously $\mathrm{dC}_{\mathrm{o}}^{\prime} / \mathrm{dt}=0$.

But from $A 3^{\prime} \frac{\mathrm{dlnN} v}{d t}+\frac{d \ln \overline{r^{3}}}{d t}=\frac{\overline{3 r^{3} v}}{\overline{r^{3}}} ;$ therefore B6 yields

$$
\frac{4 \pi C_{o} \gamma \Omega L^{2}}{3 r^{2} v}=-\frac{d l n N}{d\left(C_{1}-C_{o}\right) k T}=\frac{4 \pi C_{o} \gamma \Omega L^{2}}{\left(C_{1}-C_{o}\right) k T}\left(\frac{\overline{\partial v}}{\partial r}\right)
$$

and $\overline{r^{2} v}>0$ as expected.

We may take this opportunity and show that $v$ cannot be represented by a mean field approximation. For a diffusion controlled process in mean field approximation

$$
v=\frac{\alpha}{r}\left(\frac{1}{r *}-\frac{1}{r}\right)
$$

where $\alpha$ is a function of temperature only and $r^{*}(t)$ is the radius of the particles which, at time $t$, neither shrink nor expand. Inserting this expression in $B 7$ after rather simple manipulations, we obtain

$$
\frac{\bar{r}}{r^{*}}-1=K\left[2\left(\frac{\overline{1}}{r^{3}}\right)-\frac{1}{r^{*}}\left(\frac{1}{r^{2}}\right)\right]
$$

where $K=\frac{4 \pi C_{0} \gamma \Omega L^{2}}{3\left(C_{1}-C_{0}\right) k T}$.

Solving equation B9 for $r^{*}$, we obtain

$$
r *=\frac{\bar{r}+k\left(\frac{\overline{1}}{r^{2}}\right)}{1+2 k\left(\frac{\overline{1}}{r^{3}}\right)}
$$

As $\mathrm{K}$ is of the order of $10^{-6}$ and $\left(\frac{\overline{1}}{\mathrm{r}^{2}}\right)$ is much higher

$$
r^{*} \cong\left(\frac{1}{r^{2}}\right) / 2\left(\frac{\overline{1}}{r^{3}}\right)
$$


From B8 we easily obtain

$$
\frac{\partial v}{\partial r}=\frac{\alpha}{r^{2}}\left(\frac{2}{r}-\frac{1}{r^{*}}\right)
$$

and

$$
\left(\frac{\partial v}{\partial r}\right)=\alpha\left[2\left(\frac{\overline{1}}{r^{3}}\right)-\left(\frac{\overline{1}}{r^{2}}\right) \frac{1}{r^{*}}\right]
$$

Inserting Bio into this expression, we obtain

$$
\left(\frac{\overline{\partial v}}{\partial \mathbf{r}}\right)=0
$$

which of course is impossible in our theory. Thus we have shown that the rate of particle radius change $v$ is inadequately represented by mean field approximation. 


\section{REFERENCES}

1. G. W. Greenwood, Acta Met $\underline{4}, 243$ (1956).

2. I. M. Lifshitz and V.'V. Slyozov, J. Phys. Chem. Solids 19, 35 (1961).

3. C. Wagner, Zeit. f. Elektrochemie, 65, 581 (1961).

4. S. R. DeGroot, Thermodynamics of Irreversible Processes, Interscience Publishers, Inc., New York, 1952. 